\title{
Molecular Imaging Insights into Neurodegeneration: Focus on $\alpha$-Synuclein Radiotracers
}

\author{
Maliha Shah ${ }^{1}$, John Seibyl ${ }^{2}$, Anna Cartier $^{3}$, Ram Bhatt ${ }^{3}$, and Ana M. Catafau ${ }^{1}$ \\ ${ }^{I}$ Department of Clinical Research and Development-Neurosciences, Piramal Imaging GmbH, Berlin, Germany; ${ }^{2}$ Institute for \\ Neurodegenerative Disorders, New Haven, Connecticut; and ${ }^{3}$ ICB International, Inc., La Jolla, California
}

\begin{abstract}
Neurodegenerative diseases characterized by the presence of a-synuclein-a hallmark of pathologic inclusions termed Lewy bodies-include Parkinson's disease, dementia with Lewy bodies, and multiple-system atrophy. Although motor symptoms are related to the altered presynaptic dopaminergic function in these diseases, the appearance of $a$-synuclein inclusions precedes the involvement of the nigrostriatal dopaminergic pathway. Hence, the most accurate and earliest definition of premotor Parkinson's disease ought to rely on imaging a-synuclein rather than dopaminergic changes. Moreover, dopaminergic imaging has been controversial in monitoring the effects of investigational disease-modifying drugs. For these clinical trials, intense interest in longitudinally imaging a-synuclein as the primary pathologic process has led to efforts toward developing a suitable radiotracer for this key protein. An overview of the present $a-s y n u c l e i n$ radiotracer development scenario is presented here.
\end{abstract}

Key Words: a-synuclein; radiotracer; imaging; neurodegeneration; Parkinson's

J Nucl Med 2014; 55:1397-1400

DOI: 10.2967/jnumed.113.136515

\begin{abstract}
Alpha-synuclein is the defining component of the hallmark pathologic inclusions termed Lewy bodies (LBs), which are characteristic of Parkinson's disease (PD) and dementia with Lewy bodies (DLB), as well as being the major constituent of the glial cytoplasmic inclusions (GCIs) typical of multiple-system atrophy (MSA). In fact, the presence of intracellular $\alpha$-synuclein aggregates in multiple neurodegenerative diseases has led to their being cumulatively referred to as synucleinopathies. Various roles of $\alpha$-synuclein have been implicated in neurodegeneration, such as an involvement in mitochondrial dysfunction, proteasomal dysfunction, and vesicle trafficking within presynaptic dopaminergic neurons (1). Abnormal $\alpha$-synuclein function may affect normal dopaminergic release, resulting in decreased synaptic dopaminergic levels that may contribute to Parkinson's symptoms. Several mutations identified in the $\alpha$-synuclein locus have linked the protein to familial autosomal-dominant PD. However, most PD cases are sporadic $(>90 \%)$ and thus require another

Received May 16, 2014; revision accepted Jul. 21, 2014.

For correspondence or reprints contact: Ana M. Catafau, Piramal Imaging $\mathrm{GmbH}, \mathrm{SC}$ Trade Center 1, Avda. Corts Catalanes 5-7, Office 14, 08173 Sant

Cugat del Valles, Barcelona, Spain.

E-mail: ana.catafau@piramal.com

Published online Aug. 4, 2014.

COPYRIGHT (C 2014 by the Society of Nuclear Medicine and Molecular Imaging, Inc.
\end{abstract}

means of early and selective detection in the absence of useful genotyping.

\section{CLINICAL NEED FOR a-SYNUCLEIN TRACER}

Despite the efficacy of dopamine-replacement treatments in ameliorating motor symptoms, the progression of PD remains unaltered. Therefore, treatments designed to either slow down disease progression or entirely reverse changes are the new focus of research. Patients with the most to salvage in terms of dopaminergic cell loss will likely be those who gain the most benefit from disease modification strategies. Thus, efforts to use biomarkers to identify those PD patients most eligible for treatment have placed great emphasis on early and accurate diagnosis (2). Imaging biomarkers using both PET and SPECT have been successfully used to demonstrate clinical algorithms for identifying eligible PD patients at the early stages of motor impairment (3-5), before clinically detectable motor symptoms.

Indeed, the concept of clinical premotor PD has been developed to reflect the earliest, nonspecific, and nonmotor manifestations of PD pathology (6). Braak staging of PD brain suggests that dopaminergic loss occurs at stage III, well after the initial onset of LB appearance; hence, nigrostriatal dopaminergic tract involvement is preceded by LB formation in key areas underlying certain premotor Parkinson's symptoms, for example, loss of olfactory acuity (7-9). It has been estimated that years of occult $\alpha$-synuclein deposition may be occurring before the onset of motor symptoms; hence, the most accurate and earliest definition of premotor PD might better rely on imaging $\alpha$-synuclein rather than dopaminergic changes (8).

In addition, although theoretic, it is possible that $\alpha$-synuclein imaging would serve as the better biomarker of disease status, reflecting the key pathologic insult to the brain, which may change over the course of serial imaging. In fact, studies of PD progression in clinical therapeutic trials using dopaminergic imaging biomarkers have been criticized for the poor correlation with clinical status (10, 11). Although there are many potential reasons for this, the fact that dopamine cell loss is a downstream effect of $\alpha$-synuclein deposition is one compelling driver of interest in imaging this protein longitudinally in disease-modifying PD therapeutic trials.

\section{CHARACTERISTICS OF IDEAL $\boldsymbol{\alpha}$-SYNUCLEIN TRACER}

For a tracer to be able to recognize pathologic $\alpha$-synuclein sensitively and specifically, it must fulfill various criteria (Fig. 1). First, $\alpha$-synuclein is detected in the pathologic human brain in various forms, including diverse aggregates, fibrillar inclusions, soluble and insoluble oligomers, and pore-like species $(12,13)$. 


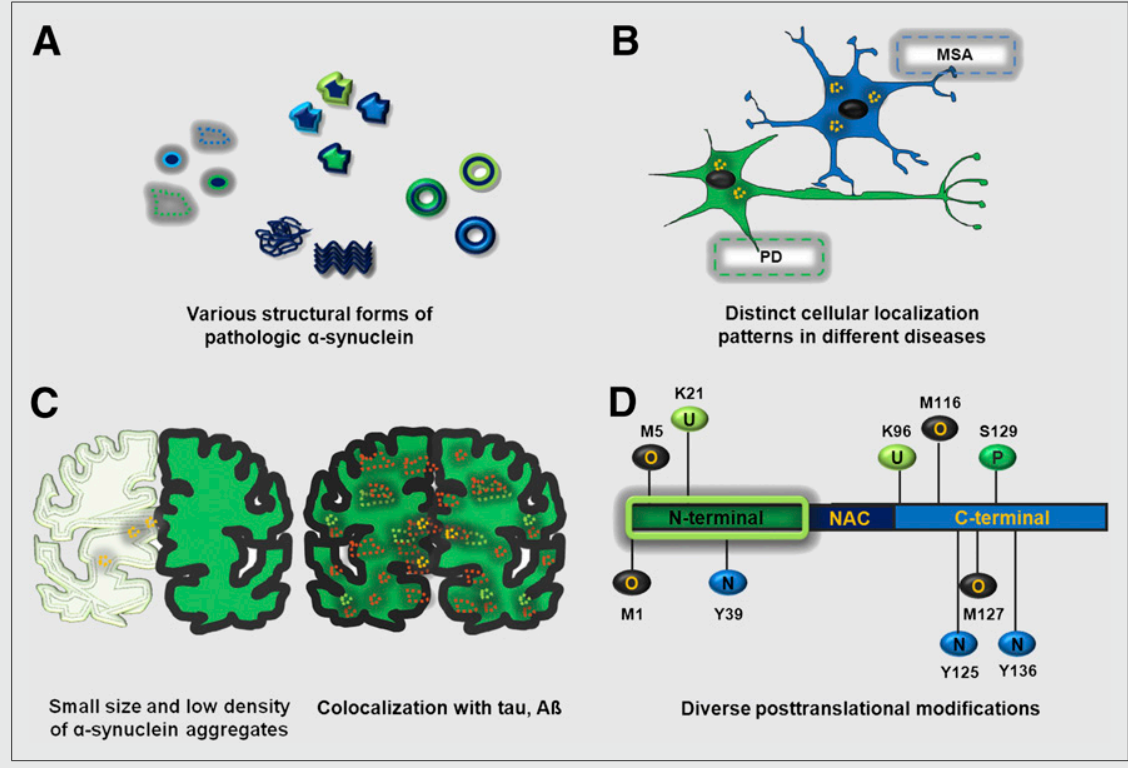

FIGURE 1. Characteristics of ideal a-synuclein tracer. Tracer should be able to recognize (anticlockwise from left) focal and diffuse aggregates, unstructured and $\beta$-pleated sheet fibrillar aggregates, annular/pore-like species, and soluble and insoluble oligomers (A); a-synuclein aggregates (yellow punctata) present in neurons (e.g., in PD) and in glia (e.g., in MSA) (B); a-synuclein aggregates despite their small size and low density in brain and a-synuclein even when it is colocalized with other proteins, for example, tau and $A \beta(C)$; multiple posttranslational modifications undergone by a-synuclein-for example, phosphorylation $(P)$ at serine-129; nitration $(N)$ of tyrosines 39,125 , and 136; ubiquitination $(U)$ of lysines 21 and 96; oxidation $(\mathrm{O})$ of all 4 methionines 1, 5, 116, and 127; and acetylation of the $\mathrm{N}$-terminal (shadowed green box) (D). NAC = nonamyloid component.
Second, different synucleinopathies are marked by differential locations of $\alpha$-synuclein aggregates, such that intraneuronal aggregates (e.g., LBs) are typical of PD, whereas oligodendrocytic aggregates (e.g., GCIs) are typical of MSA, both of which should be detected by the optimal tracer, thereby providing the potential for differential diagnosis. Third, although $\alpha$-synuclein is highly abundant in the nervous system, accounting for $1 \%$ of total cytosolic protein (14), aggregates of $\alpha$-synuclein have a relatively low density in the diseased brain and additionally have a typically small size, complicating detection. Furthermore, $\alpha$ synuclein is often seen to be colocalized with other aggregating proteins, for example, tau and amyloid- $\beta$ (A $\beta)(15)$, as well as a variety of other proteins accumulated within cellular inclusions, for example, synphilin-1 and parkin. Hence, the said tracer must be able to specifically detect $\alpha$-synuclein with regard to other codepositing members. Fourth, $\alpha$-synuclein is known to undergo diverse posttranslational modifications, all of which should be detectable by the tracer. For instance, synucleinopathies are usually associated with inflammation and elevated levels of oxidative stress

TABLE 1

Summary of Characteristics of PET Radiotracers Relevant to a-Synuclein (Syn) Imaging

Ligand

*Values determined for ${ }^{3} \mathrm{H}$-PIB.

${ }^{\dagger}$ Fibrils used in assay were $A \beta_{1-40}$.

$\mathrm{K}_{\mathrm{d}}=$ dissociation constant. 
in affected brain areas, which favor oxidative modifications of $\alpha$-synuclein, such as the nitration of tyrosine residues, and methionine sulfoxidations, both of which are observed in postmortem PD brain sections (16). Furthermore, phosphorylated $\alpha$-synuclein immunoreactive for ubiquitin has been detected in both LBs and GCIs (17), whereas a ubiquitous N-terminal acetylation was detected in LBs from DLB patients (18).

In addition, there needs to be adequate brain penetration; good washout of nonspecific uptake permitting good signal-to-noise contrast; lack of P-glycoprotein substrate activity; lack of radioactive metabolites; and properties amenable to determining an appropriate quantitative and reproducible outcome measure, preferably a simple, noninvasive tissue-to-blood or tissue-to-reference region ratio. Thus, the optimal $\alpha$-synuclein tracer must be able to address all of the above factors to provide accurate and comprehensive in vivo detection of pathologic $\alpha$-synuclein. Although this task appears to be rather daunting, various efforts have been made toward adopting existing amyloid tracers for $\alpha$-synuclein detection and developing novel molecules of promising potential, as described below.

\section{OVERVIEW OF $\alpha$-SYNUCLEIN PET TRACERS}

This section focuses on some $\alpha$-synuclein PET tracers in active development-namely, ${ }^{11} \mathrm{C}$-Pittsburgh compound-B (PIB), ${ }^{18} \mathrm{~F}-$ BF227, and ${ }^{125}$ I-SIL23-demonstrating the principles described in the section above. The PET tracer PIB was originally developed

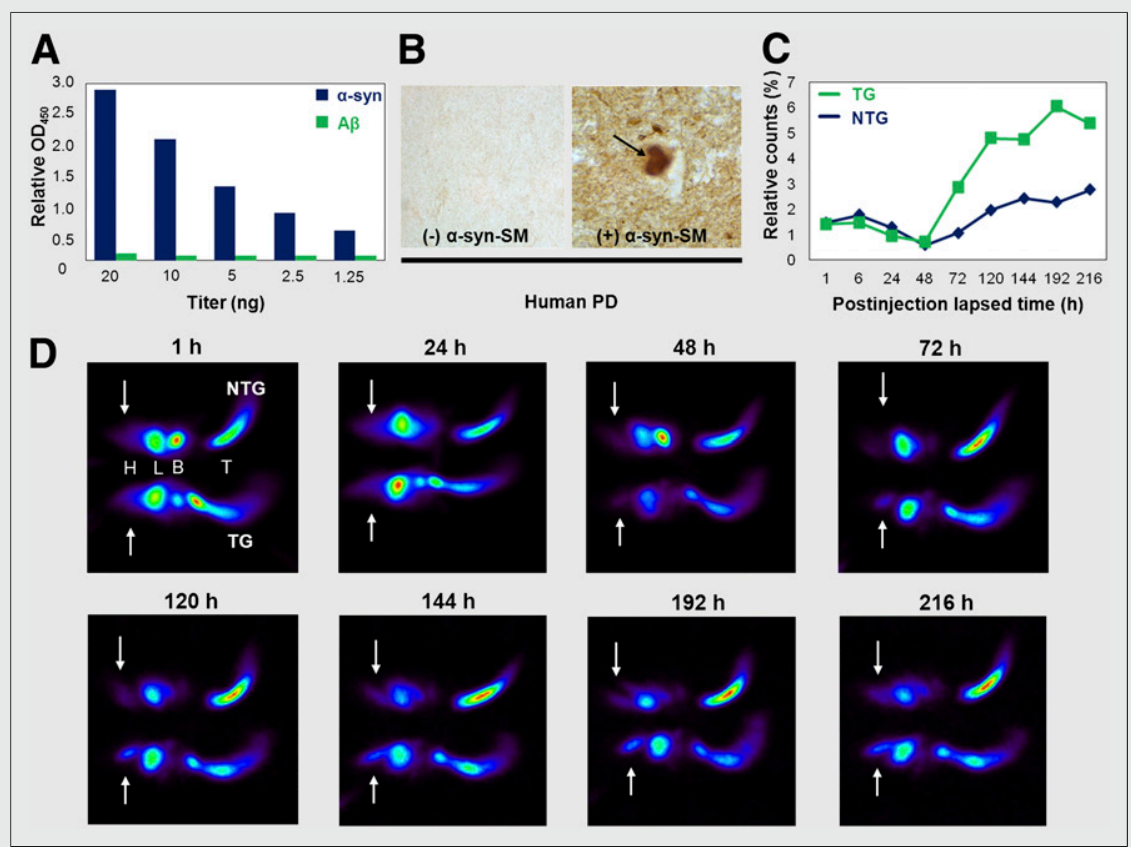

FIGURE 2. Detection of a-synuclein by ${ }^{125}$ - $a$-synuclein-SM. (A) Binding specificity: enzymelinked immunosorbent assay comparing ${ }^{125}$-a-synuclein-SM binding to recombinant human a-synuclein and $A \beta$. (B) Tissue specificity of ${ }^{125} \mathrm{I}$ - $\mathrm{a}$-synuclein-SM tested on human PD brain sections. Arrow indicates Lewy body. (C) Biodistribution of ${ }^{125} \mathrm{I}$ - $\mathrm{a}$-synuclein-SM in a-synuclein transgenic (TG) and nontransgenic (NTG) mice expressed as ratio of relative radioactivity counts between head and whole body. Radioactivity in all organs was measured by $y$ counter. (D) In vivo imaging of ${ }^{125} \mathrm{I}$-a-synuclein-SM: approximately $50 \mu \mathrm{g}$ were injected in TG and NTG mice and images acquired over time. Amount available for circulation was probably less than $50 \mu \mathrm{g}$ because of aggregate formation after labeling and binding to tail vein. Signal detected from planar scintigraphy in NTG mice is not due to unspecific binding but to cross-reaction of ${ }^{125}$ - $\alpha$-synuclein-SM with both human and murine $a$-synuclein. Arrows indicate brain. $\mathrm{B}=$ bladder; $\mathrm{H}=$ head; $\mathrm{L}=$ liver; $\mathrm{T}=$ tail. to target $A \beta$ plaques, but because it is a derivative of thioflavin $T$, which stains essentially all types of amyloid, it is conceivable that PIB may also bind to another amyloidogenic protein, that is, $\alpha$-synuclein. However, the preclinical data on the utility of PIB as an $\alpha$-synuclein-specific PET tracer appear to be rather inconsistent (19). Although there is some evidence of PIB binding to aggregated $\alpha$-synuclein in vitro and in vivo, its much higher afpossible that future compounds structurally related to PIB may 政 particular, BF227. In vitro ${ }^{18} \mathrm{~F}-\mathrm{BF} 227$ saturation studies the $A \beta_{1-42}$ fibrils indicated that ${ }^{18} \mathrm{~F}-\mathrm{BF} 227$ abeled both $\mathrm{A} \beta$ plaques and LBs in immunohistochemical/ fluorescence analyses of $\mathrm{AD}$ and $\mathrm{PD}$ brain sections, respectively. and detect $\alpha$-synuclein deposits in the living brains of 8 MSA the subcortical white matter, globus pallidus, substantia nigra, and other regions were higher in MSA subjects than in controls, which was coincident with GCI-rich brain areas in MSA. However, it should be kept in mind that BF227 was initially designed as an $A \beta$ imaging agent (22); hence, both its affinity and its selectivity pose questions regarding its applicability in specific $\alpha$-synuclein detection.

A recent study identified ${ }^{125}$ I-SIL23 as a novel $\alpha$-synuclein PET radioligand (23). The molecule binds to $\alpha$-synuclein fibrils in postmortem brain tissue from PD patients as well as to $\alpha$-synuclein in a transgenic mouse model for PD. The density of SIL23 binding sites correlated with the level of fibrillar $\alpha$-synuclein in PD brain tissue and was high enough to enable in vivo imaging with high-affinity ligands. However, the affinity of SIL23 for $\alpha$-synuclein may not be optimal for imaging fibrillar $\alpha$-synuclein in vivo (Table 1). Furthermore, high nonspecific binding, including nonspecific binding in white matter likely secondary to lipophilic interactions, also appears to limit autoradiography with SIL23 in preliminary experiments.

In conclusion, whereas progress has been made toward the identification of a suitable $\alpha$-synuclein PET tracer, an ideal candidate still remains elusive.

\section{CHALLENGES AND FUTURE DIRECTIONS}

Despite the challenges in developing a successful $\alpha$-synuclein PET tracer, there are several promising leads under investigation, 
including both small molecules and antibodies. The latter are particularly interesting insofar as antibodies or antibody fragments directed at pathogenic proteins implicated in neurodegenerative disorders have been suggested as one therapeutic route to modify disease progression. It is possible to label these for PET and SPECT imaging, although the protracted kinetics require longer half-life isotopes.

Recently, an approximately 40-kDa molecule having Specific Molecular Architecture for Recognition and Therapy (SMART) has been generated which detects $\alpha$-synuclein with high specificity ( $\alpha$-synuclein-SM). One study examining ${ }^{125} \mathrm{I}-\alpha$-synuclein-SM in PDlike transgenic (TG) and nontransgenic (NTG) mice demonstrated higher brain uptake and retention in the TG animals than in controls (Fig. 2). ${ }^{125} \mathrm{I}-\alpha$-synuclein-SM continued to accumulate in the TG brain for at least 9 days. Furthermore, biodistribution analysis of ${ }^{125} \mathrm{I}-\alpha$-synuclein-SM showed approximately $15 \%$ higher brain penetration relative to blood in the TG mice. Ongoing research will ultimately prove whether this strategy is fruitful toward producing a viable $\alpha$-synuclein imaging agent.

The importance of this brain target will continue to fuel efforts in this arena, and studies are ongoing to develop additional small molecules targeting $\alpha$-synuclein as well.

\section{CONCLUSION}

$\alpha$-synuclein is a target of great importance in the pathophysiology of PD and other neurodegenerative disorders, as well as their potential treatment. Up to this point, no viable radiotracer has been generated for the detection of brain $\alpha$-synuclein. A large scientific effort is currently under way to develop this, with efforts directed toward traditional labeled small molecules as well as antibodies or partial antibodies. It remains to be seen what role the successful $\alpha$-synuclein radiotracer could serve and whether there are potential advantages from the standpoint of earlier and accurate diagnosis, eligibility assessment for clinical trials, and disease monitoring of therapeutics in both research and clinical contexts.

\section{DISCLOSURE}

Maliha Shah and Ana Catafau are Piramal Imaging GmbH employees. Dr. Seibyl has equity interest in Molecular Neuroimaging, LLC. No other potential conflict of interest relevant to this article was reported.

\section{ACKNOWLEDGMENTS}

We gratefully acknowledge Dr. Heribert Schmitt-Willich and Jörg Jannsen for assistance with chemical structures and figures, respectively.

\section{REFERENCES}

1. Burré J, Sharma M, Tsetsenis T, Buchman V, Etherton MR, Sudhof TC. Alphasynuclein promotes SNARE-complex assembly in vivo and in vitro. Science. 2010;329:1663-1667.

2. Seibyl J, Russell D, Jennings D, Marek K. Neuroimaging over the course of Parkinson's disease: from early detection of the at-risk patient to improving pharmacotherapy of later-stage disease. Semin Nucl Med. 2012;42:406-414.
3. Seibyl J, Russell D, Jennings D, Marek K. The molecular basis of dopaminergic brain imaging in Parkinson's disease. Q J Nucl Med Mol Imaging. 2012;56: $4-16$.

4. Berg D, Marek K, Ross GW, Poewe W. Defining at-risk populations for Parkinson's disease: lessons from ongoing studies. Mov Disord. 2012;27: 656-665.

5. Iranzo A, Lomena F, Stockner H, et al. Decreased striatal dopamine transporter uptake and substantia nigra hyperechogenicity as risk markers of synucleinopathy in patients with idiopathic rapid-eye-movement sleep behaviour disorder: a prospective study. Lancet Neurol. 2010;9:1070-1077.

6. Marek K, Jennings D. Can we image premotor Parkinson disease? Neurology. 2009;72(suppl):S21-S26.

7. Hawkes CH, Del Tredici K, Braak H. A timeline for Parkinson's disease. Parkinsonism Relat Disord. 2010;16:79-84.

8. Dickson DW, Braak H, Duda JE, et al. Neuropathological assessment of Parkinson's disease: refining the diagnostic criteria. Lancet Neurol. 2009;8: 1150-1157.

9. Braak H, Del Tredici K. Neuroanatomy and pathology of sporadic Parkinson's disease. Adv Anat Embryol Cell Biol. 2009;201:1-119.

10. Fahn S, Oakes D, Shoulson I, et al. Levodopa and the progression of Parkinson's disease. N Engl J Med. 2004;351:2498-2508.

11. Brooks DJ, Frey KA, Marek KL, et al. Assessment of neuroimaging techniques as biomarkers of the progression of Parkinson's disease. Exp Neurol. 2003;184 (suppl 1):S68-S79.

12. Paleologou KE, Kragh CL, Mann DM, et al. Detection of elevated levels of soluble alpha-synuclein oligomers in post-mortem brain extracts from patients with dementia with Lewy bodies. Brain. 2009;132:1093-1101.

13. Choi JH, Stubblefield B, Cookson MR, et al. Aggregation of alpha-synuclein in brain samples from subjects with glucocerebrosidase mutations. Mol Genet Metab. 2011;104:185-188.

14. Stefanis L. $\alpha$-Synuclein in Parkinson's disease. Cold Spring Harb Perspect Med. 2012;2:a009399.

15. Kotzbauer PT, Cairns NJ, Campbell MC, et al. Pathologic accumulation of alphasynuclein and $\mathrm{A} \beta$ in Parkinson disease patients with dementia. Arch Neurol. 2012;69:1326-1331.

16. Schildknecht S, Gerding HR, Karreman C, et al. Oxidative and nitrative alphasynuclein modifications and proteostatic stress: implications for disease mechanisms and interventions in synucleinopathies. J Neurochem. 2013;125:491-511.

17. Uchihara T, Nakamura A, Mochizuki Y, et al. Silver stainings distinguish Lewy bodies and glial cytoplasmic inclusions: comparison between Gallyas-Braak and Campbell-Switzer methods. Acta Neuropathol. 2005;110:255-260.

18. Anderson JP, Walker DE, Goldstein JM, et al. Phosphorylation of Ser-129 is the dominant pathological modification of alpha-synuclein in familial and sporadic Lewy body disease. J Biol Chem. 2006;281:29739-29752.

19. Maetzler W, Reimold M, Liepelt I, et al. $\left[{ }^{11} \mathrm{C}\right] \mathrm{PIB}$ binding in Parkinson's disease dementia. Neuroimage. 2008;39:1027-1033.

20. Fodero-Tavoletti MT, Mulligan RS, Okamura N, et al. In vitro characterisation of BF227 binding to alpha-synuclein/Lewy bodies. Eur J Pharmacol. 2009;617: 54-58.

21. Kikuchi A, Takeda A, Okamura N, et al. In vivo visualization of alpha-synuclein deposition by carbon-11-labelled 2-[2-(2-dimethylaminothiazol-5-yl)ethenyl]-6[2-(fluoro)ethoxy]benzoxazole positron emission tomography in multiple system atrophy. Brain. 2010;133:1772-1778

22. Tomita N, Furukawa K, Okamura N, et al. Brain accumulation of amyloid beta protein visualized by positron emission tomography and BF-227 in Alzheimer's disease patients with or without diabetes mellitus. Geriatr Gerontol Int. 2013;13:215-221.

23. Bagchi DP, Yu L, Perlmutter JS, et al. Binding of the radioligand SIL23 to alphasynuclein fibrils in Parkinson disease brain tissue establishes feasibility and screening approaches for developing a Parkinson disease imaging agent. PLoS ONE. 2013;8:e55031.

24. Fodero-Tavoletti MT, Smith DP, McLean CA, et al. In vitro characterization of Pittsburgh compound-B binding to Lewy bodies. J Neurosci. 2007;27:1036510371.

25. Ye L, Velasco A, Fraser G, et al. In vitro high affinity alpha-synuclein binding sites for the amyloid imaging agent PIB are not matched by binding to Lewy bodies in postmortem human brain. J Neurochem. 2008;105:1428-1437. 